\title{
Kondo Destruction in Heavy Fermion Quantum Criticality and the Photoemission Spectrum of $\mathrm{YbRh}_{2} \mathrm{Si}_{2}$
}

 \\ ${ }^{a}$ Institute of Solid State Physics, Vienna University of Technology, \\ Wiedner Hauptstr. 8-10, 1040 Vienna, Austria \\ ${ }^{b} \mathrm{HH}$ Wills Laboratory, University of Bristol, Bristol BS8 1TH, UK \\ ${ }^{c}$ Max Planck Institute for Chemical Physics of Solids, Nöthnitzer Str. 40, \\ 01187 Dresden, Germany \\ ${ }^{d}$ Center for Correlated Matter, Zhejiang University, Hangzhou, Zhejiang 310058, China \\ ${ }^{e}$ Department of Physics and Astronomy, Rice University, Houston, Texas 77005, USA
}

\begin{abstract}
Heavy fermion metals provide a prototype setting to study quantum criticality. Experimentally, quantum critical points have been identified and studied in a growing list of heavy fermion compounds. Theoretically, Kondo destruction has provided a means to characterize a class of unconventional quantum critical points that goes beyond the Landau framework of orderparameter fluctuations. Among the prominent evidence for such local quantum criticality have been measurements in $\mathrm{YbRh}_{2} \mathrm{Si}_{2}$. A rapid crossover is observed at finite temperatures in the isothermal field dependence of the Hall coefficient and other transport and thermodynamic quantities, which specifies a $T^{*}(B)$ line in the temperature $(T)$-magnetic field $(B)$ phase diagram. Here, we discuss what happens when temperature is raised, by analyzing the ratio of the crossover width to the crossover position. With this ratio approaching unity at $T \gtrsim 0.5 \mathrm{~K}, \mathrm{YbRh}_{2} \mathrm{Si}_{2}$ at zero magnetic field belongs to the quantum-critical fluctuation regime, where the single-particle spectral function has significant spectral weight at both the small and large Fermi surfaces. This implies that, in this temperature range, any measurements sensitive to the Fermi surface will also see a significant spectral weight at the large Fermi surface. The angle-resolved photoemission spectroscopy (ARPES) experiments recently reported for $\mathrm{YbRh}_{2} \mathrm{Si}_{2}$ at $T>1 \mathrm{~K}$ are consistent with this expectation, and therefore support the association of the $T^{*}(B)$ line with the
\end{abstract}


physics of Kondo destruction.

Keywords: quantum criticality, heavy fermion metals, Kondo destruction, ARPES

\section{Introduction}

Quantum criticality is of extensive current interest in a variety of strongly correlated electron systems [1, 2]. Heavy fermion metals have emerged as prototype systems for quantum criticality [3, 4, 5]. The small energy scales of these materials lead to increased tunability of the ground state by parameters such as pressure, magnetic field or chemical substitution. This facilitates the realization of quantum critical points (QCPs). Indeed, QCPs have been experimentally observed in a considerable number of antiferromagnetic heavy fermion metals.

Heavy fermion metals have provided a setting to explore quantum criticality that goes beyond the conventional Landau type of order-parameter fluctuations [6]. Theoretically, an unconventional type of quantum criticality has been advanced, which is characterized by a critical destruction of the Kondo effect [7, 8]. The Kondo destruction is manifested through a jump of the Fermi surface at zero temperature, from small (i.e., not incorporating the $f$-electrons) to large (involving the $f$-electrons).

Evidence for this local quantum criticality has come from a variety of experiments. For example, inelastic neutron scattering experiments near the Au-substitution induced QCP in $\mathrm{CeCu}_{6}$ [9] and the Pd-substitution induced QCP in $\mathrm{UCu}_{5}$ [10] found the type of dynamical scaling consistent with the theory. Moreover, de Haas-van Alphen measurements [1] showed a jump from a small to a large Fermi surface as pressure is increased through the antiferromagnetic QCP of CeRhIn 5 [12, 13].

Measurements across the magnetic field-induced QCP in $\mathrm{YbRh}_{2} \mathrm{Si}_{2}$ have revealed a rapid crossover in the isothermal field dependence of the Hall coefficient and other magnetotransport and thermodynamic quantities [14, 15, 16, 17. These studies specify a $T^{*}(B)$ line in the temperature-magnetic field phase diagram. Extrapolating this crossover behavior towards lower temperatures has led to the conclusion that, in the zero-temperature limit, the Hall coefficient and several other transport and thermodynamic properties display a sudden jump across the critical field.

In this paper, we discuss the implications of the above understandings for 
ARPES, which can only be carried out at zero magnetic field and is presently limited to relatively high temperatures. Towards this goal, we discuss what happens to the isothermal crossover as the temperature is raised, by analyzing the ratio of the crossover width to the crossover position. The resulting temperature dependence of this ratio shows that, for $T \gtrsim 0.5 \mathrm{~K}, \mathrm{YbRh}_{2} \mathrm{Si}_{2}$ at zero magnetic field fully belongs to the quantum-critical fluctuation regime. In this regime, the single-particle spectral function has significant spectral weight at both the small and the large Fermi surface. This implies that ARPES measurements in this temperature range are expected to also see a significant spectral weight at the large Fermi surface. The ARPES experiments recently reported by Kummer et al. [19] on $\mathrm{YbRh}_{2} \mathrm{Si}_{2}$ at $T \gtrsim 1 \mathrm{~K}$ are consistent with the above expectation.

\section{Kondo effect and its critical destruction}

Magnetic heavy fermion metals contain a lattice of local moments that are antiferromagnetically coupled to the spins of a conduction electron band. Usually, the transition is between an antiferromagnetically ordered phase and the paramagnetic metal phase.

Within the Landau framework, quantum criticality is described in terms of the fluctuations of the antiferromagnetic order parameter. The metallic nature would be manifested only through the presence of Landau damping of the order parameter field by a decay into particle-hole excitations. This is the picture of a spin-density-wave (SDW) QCP [20, 21, 22]. The effect of higher order terms in the coupling between the order parameter and gapless conduction electrons is the subject of continued theoretical interest.

However, the paramagnetic ground state of the heavy fermion metals involves the lattice Kondo effect, with a nonzero amplitude for the Kondo singlet, i.e., entanglement between the local moments and the spins of the conduction electrons. At a local QCP, this Kondo-singlet amplitude goes to zero continuously as the QCP is approached from the paramagnetic side [7, 23, 24, as the antiferromagnetic order sets in. This is illustrated in Fig.11. top panel. Here, $E_{l o c}^{*}$ describes the energy scale for the Kondo destruction. As the control parameter $\delta$ approaches $\delta_{c}$ from the paramagnetic side $\left(\delta>\delta_{c}\right)$, $E_{l o c}^{*}$ vanishes at $\delta_{c}$, where the Néel order smoothly sets in.

The critical destruction of the Kondo effect affects the scaling of the order-parameter dynamics. The dynamical spin susceptibility at the QCP 

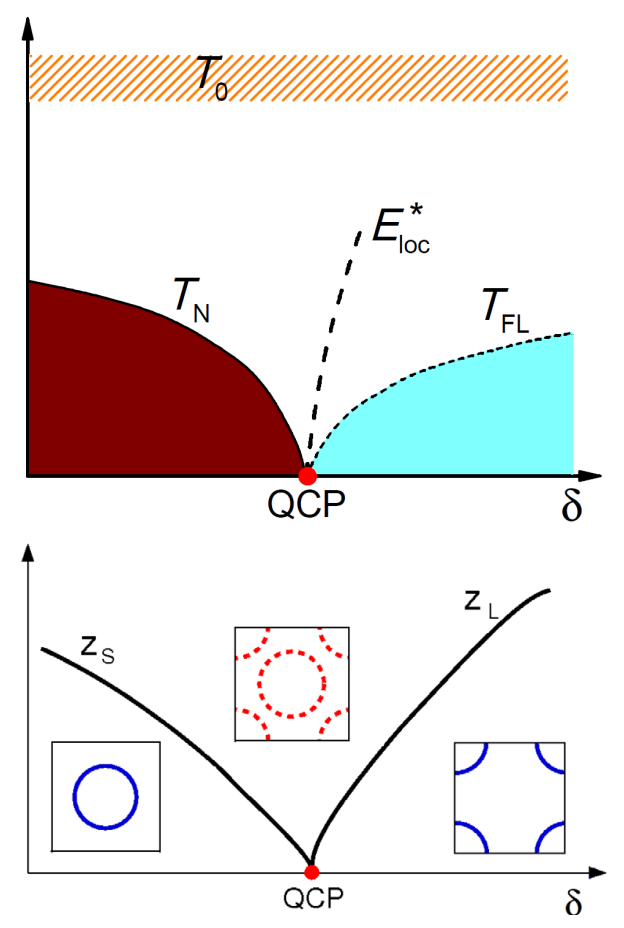

Figure 1: Top: Kondo destruction at a heavy fermion QCP. Bottom: Small (left) and large (right) Fermi surfaces (from [18]). Each Fermi surface is marked by a solid line, which denotes a nonzero quasiparticle spectral weight $z_{S}$ or $z_{L}$. In the quantum critical regime, while both $z_{S}$ and $z_{L}$ vanish, low-energy single-particle excitations occur at both the small and large Fermi surfaces and have a non-Fermi liquid form.

was shown to have the following form:

$$
\chi(\mathbf{q}, \omega)=\frac{1}{f(\mathbf{q})+A(-i \omega)^{\alpha} W(\omega / T)} .
$$

While the form itself has been derived analytically (within an $\epsilon$-expansion) 7. 24, the exponent $\alpha$ was determined numerically. It was found to be close to 0.75 (ranging from 0.72 to 0.78 depending on the method of solution) in the case of an Ising-anisotropic Kondo lattice [23, 25, 26].

In addition to the form of dynamical scaling and the extra energy scale $E_{l o c}^{*}$ vanishing at the QCP, the Kondo destruction is also manifested in the evolution of the Fermi surface across the QCP [24]. This is illustrated in Fig. 1, bottom panel. 
- For $\delta<\delta_{c}$ and at sufficiently low temperatures, the Fermi surface is small and sharp. In other words, the single particle excitations are propagating quasiparticles and gapless near the small Fermi surface, but such excitations display a small energy gap at the large Fermi surface.

- For $\delta>\delta_{c}$, again at sufficiently low temperatures, the Fermi surface is large and sharp. In other words, the single particle excitations are propagating quasiparticles and gapless near the large Fermi surface; such excitations have a small energy gap at the small Fermi surface.

- In the crossover region, incoherent single-particle excitations exist at both the small and the large Fermi surface. The quasiparticle residue for the large Fermi surface, $z_{L}$, as well as its counterpart for the small Fermi surface, $z_{S}$, vanish at zero energy and zero temperature; both depend on energy and temperature in a power-law fashion. In other words, the single-particle spectral weights for small but nonzero energies and temperatures are nonzero at both the small and the large Fermi surface. At the same time, the single-particle excitations assume a non-Fermi liquid form everywhere on the Fermi surfaces (leaving no "cold" portions of the Fermi surfaces).

\section{Evidence for Kondo destruction in quantum critical heavy fermion metals}

\subsection{Dynamical scaling}

Inelastic neutron scattering experiments provide a means to measure the dynamical spin susceptibility. Such experiments are challenging because they require large single crystalline samples. Nontheless, results are available in several quantum critical heavy fermion metals. The most prominent example is the Ising-anisotropic $\mathrm{CeCu}_{6-\mathrm{x}} \mathrm{Au}_{\mathrm{x}}$ at $x_{c}=0.1$, where the dynamical spin susceptibility has been found [9] to have the form of Eq. (1) and a critical exponent of about 0.75. A similar form was also found in the heavy fermion metal $\mathrm{UCu}_{5-\mathrm{x}} \mathrm{Pd}_{\mathrm{x}}[10]$.

\subsection{Fermi surface evolution}

$\mathrm{CeRhIn}_{5}$ displays a continuous quantum phase transition as pressure is raised across $p_{c}$ [12, 27], at magnetic fields (of about $10 \mathrm{~T}$ ) above $H_{c 2}$, the 


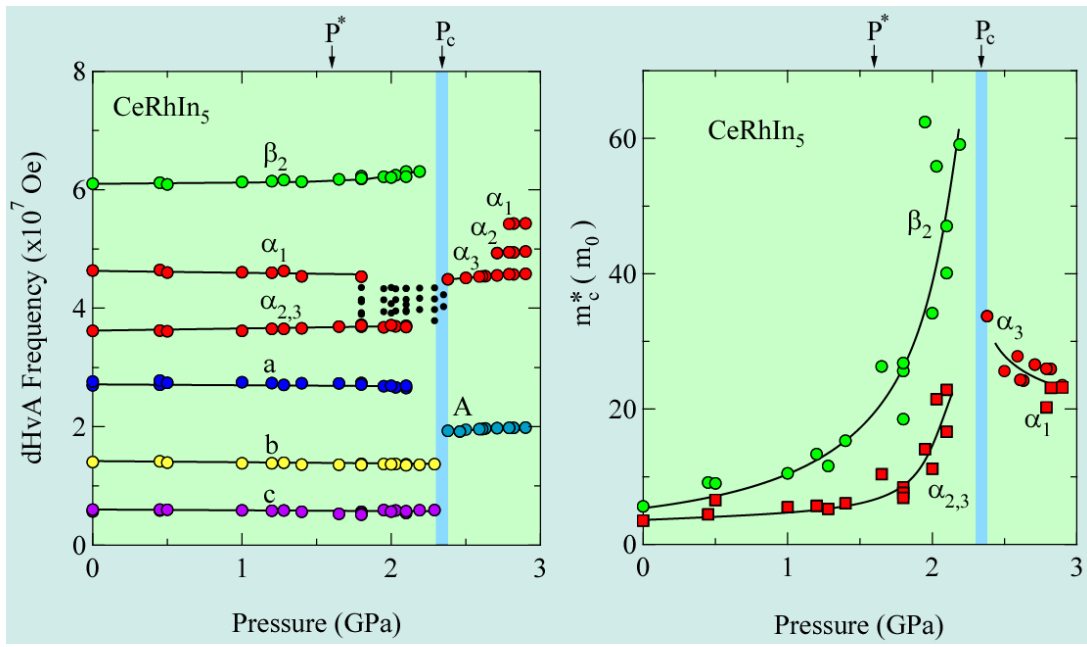

Figure 2: Jump of Fermi surface across the critical pressure in $\mathrm{CeRhIn}_{5}$, observed by dHvA measurements (adapted from [1]).

upper critical field for superconductivity. Quantum oscillations have been observed by de Haas-van Alphen (dHvA) measurements at various pressures at magnetic fields between $10 \mathrm{~T}$ and $17 \mathrm{~T}$ [11]. A jump of the Fermi surface has been evidenced by the observation that the dHvA frequencies undergo a sharp jump across $p_{c}$. The dHvA frequencies are compatible with a small Fermi surface in the antiferromagnetically ordered state at $p<p_{c}$, and with a large Fermi surface in the paramagnetic state at $p>p_{c}$. The dHvA measurements also indicate that the cyclotron mass diverges at $p_{c}$, providing evidence that the quasiparticle residues $z_{L}$ and $z_{S}$ indeed vanish at the QCP, see Fig. 1, bottom.

\section{Extrapolating the isothermal crossover to lower temperatures}

For $\mathrm{YbRh}_{2} \mathrm{Si}_{2}$, the isothermal crossovers as a function of magnetic field $B$ have been studied between $0.02 \mathrm{~K}$ and $1 \mathrm{~K}$. The pertinent measurements include magnetotransport, Hall effect and magnetoresistance, as well as thermodynamic properties, including magnetization and magnetostriction.

To draw conclusions about the nature of the QCP, the efforts have been directed towards the evolution of the isothermal crossover behavior as temperature is lowered. The lowest temperature of the studies is about $20 \mathrm{mK}$ [15]. Importantly, as temperature is lowered, the full width at half maximum 

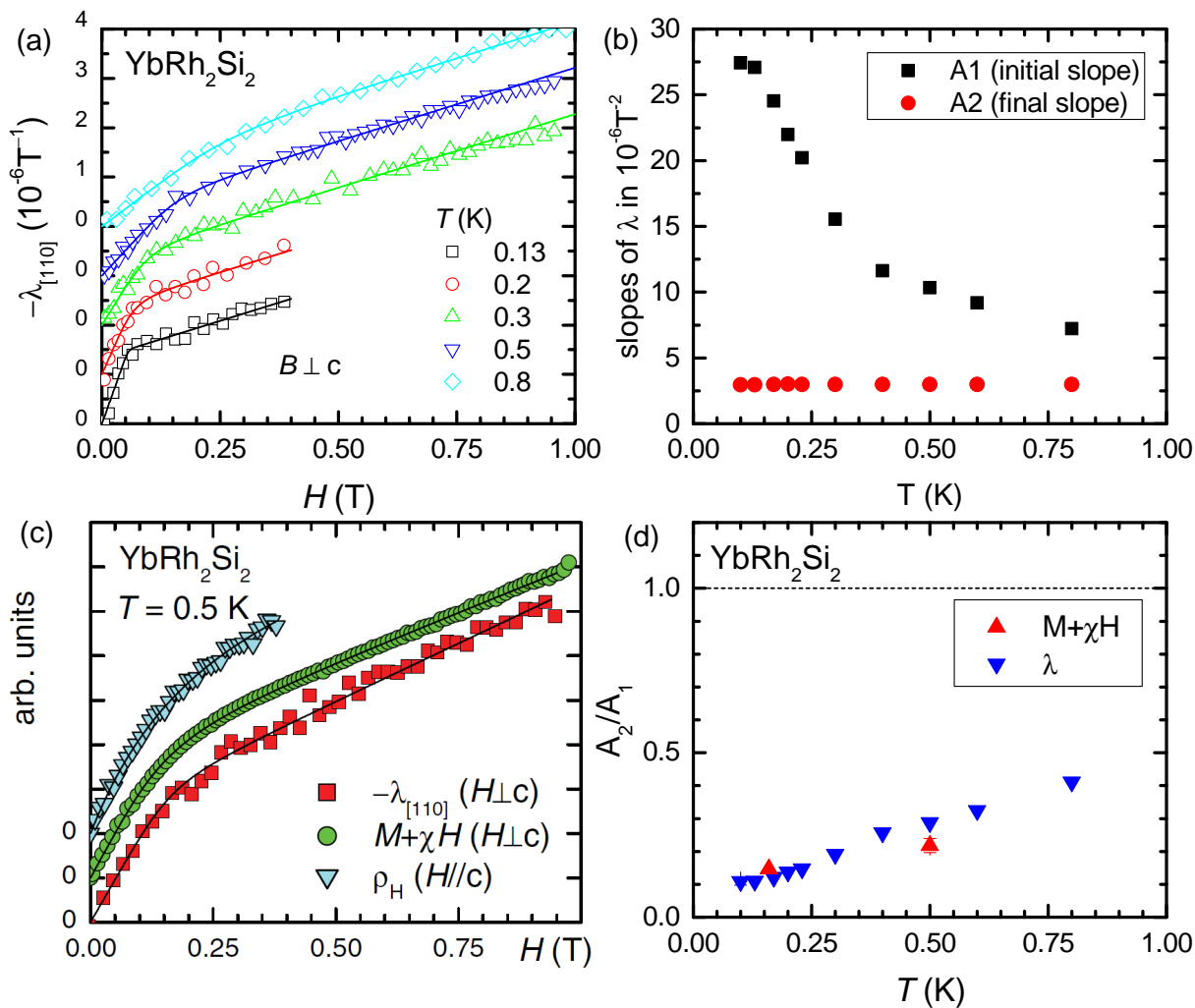

Figure 3: Thermodynamic signatures of the $T^{*}(B)$ line. (a) Isothermal magnetostriction $\lambda$ as a function of the magnetic field at selected temperatures 28. (b) Initial slope $A_{1}$ for fields below $B^{*}(T)$, and the final slope $A_{2}$ for fields above $B^{*}(T)$ as extracted from fits to the magnetostriction. The difference between the two slopes, i.e. the amplitude of the crossover, grows as temperature is reduced. (c) Isothermal magnetostriction $\lambda$, $\tilde{M}=M+\chi H$ with the magnetisation $M$ and susceptibility $\chi$, and Hall resistivity $\rho_{\mathrm{H}}$ at $0.5 \mathrm{~K}$ [15]. The magnetization $M$ vs. $\rho_{\mathrm{H}}$ behaves similarly as $\tilde{M}$ vs. $\rho_{\mathrm{H}}$ [15]. (d) The ratio of the two slopes $A_{2} / A_{1} \neq 1$ differing from 1 marks a finite crossover amplitude in the magnetostriction and $\tilde{M}[15]$.

(FWHM) of the isothermal crossover in all the measured properties decreases [14, 15, 16]. It extrapolates to zero in the limit of zero temperature, consistent with a jump of the $T=0$ Hall coefficient and related properties when the tuning parameter, the magnetic field, crosses the QCP.

An example of the crossover behavior is illustrated in Fig. 3(a), which shows the isothermal field dependence of the magnetostriction. For each 
temperature, the field dependence can be fitted in terms of a crossover function with an initial slope $A_{1}$, applicable to the regime of low fields prior to the crossover, and a final slope $A_{2}$, applicable to high fields beyond the crossover. The observed decrease of the slope $\left(\Delta A=A_{2}-A_{1}<0\right)$ corresponds to a drop in the derivative $d \lambda / d B$ of the magnetostriction isotherm. The amplitude of this drop increases as $T$ is lowered, thereby extrapolating to a nonzero value in the zero-temperature limit. Together with the vanishing width of the crossover, this implies a jump in $d \lambda / d B$. Similar behavior has also been observed for the magnetization vs. $B$ (Fig. 3(d)), and in the magnetotransport properties Hall coefficient and magnetoresistance; in the latter two, the magnitude of the drop decreases as $T$ is lowered, but extrapolates always to a non-zero value [29]. We take this as an indication that the underlying Fermi surface jump is a robust effect and its manifestations in magnetotransport are less pronounced because of the interfering effects of multiple bands that are present in $\mathrm{YbRh}_{2} \mathrm{Si}_{2}$ [16].

These studies lead to the conclusion that for $B=0$ (and $B<B_{c}$ more generally) and at sufficiently low temperatures, the Fermi surface is small and sharp. For $B>B_{c}$, again at sufficiently low temperatures, the Fermi surface is large and sharp. In the crossover region, incoherent single-particle excitations exist at both the small and the large Fermi surface.

The important question is what happens at higher temperatures.

\section{Reaching up in temperature}

One of the reasons for quantum criticality being of interest is that the unusual excitations associated with the QCP ground state govern a large parameter regime at nonzero temperatures. It is therefore of considerable interest to address how a quantum critical system behaves as we reach upwards in temperature.

For $\mathrm{YbRh}_{2} \mathrm{Si}_{2}$, the isothermal crossover described in the previous section can also be analyzed to infer the nature of the higher temperature portion of the $T-B$ phase diagram. For reasons that will become clear, we are interested in what happens at temperatures on the order of $1 \mathrm{~K}$ and higher, at $B=0$. As can be inferred from the $T-B$ phase diagram for $T \leq 0.3$ $\mathrm{K}$, shown in Fig. 4(a), for these higher temperatures the system obviously is in the "orange" quantum-critical regime even at $B=0$. The same point

can be understood more quantitatively. In Fig. 4(b), we show the ratio of the FWHM/2 associated with the isothermal crossover to $B_{\text {inf }}$, defined as the 

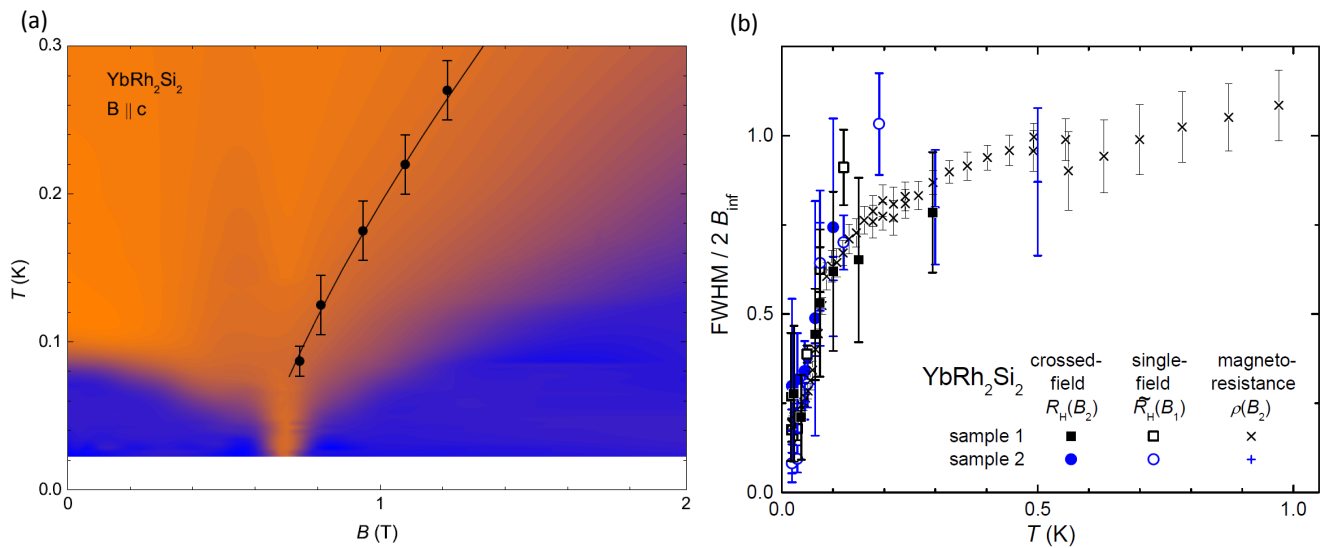

Figure 4: (a) $T-B$ phase diagram. The color code indicates the exponent $\varepsilon$ of the temperature dependent resistivity, i.e. $\rho(T)=\rho_{0}+c T^{\varepsilon}$ measured for $B \| c$ [30]. The line indicates $T^{*}(B)$, as obtained from susceptibility measurements for $B \perp c$ [28] and rescaled by a factor 11 . (b) The ratio of the FWHM/2 of the isothermal crossover to $B_{\text {inf }}$, where the magnetoresistance exhibits an inflection point with respect to $B$. As temperature is raised to about $0.5 \mathrm{~K}$ and above, this ratio reaches 1 within the error bars, implying that $B=0$ is already part of the crossover regime.

magnetic field at which the Hall coefficient and the magnetoresistance as well as the field derivatives of the magnetization and the magnetostriction exhibit an inflection point. As temperature is raised to about $0.5 \mathrm{~K}$ and above, this ratio reaches 1 within error bars. The fact that this happens at such low temperatures is tied up with the fact that $B^{*}(T)$ is relatively small, which is in turn due to the small value of the critical field, $B_{c}=B^{*}(T=0)$.

\section{Comments on the recent ARPES measurements}

\subsection{ARPES in $Y b R h_{2} S i_{2}$}

Our considerations above show that for $T \gtrsim 0.5 \mathrm{~K}$ the width of the isothermal crossover is sufficiently large to make even $B=0$ to fall in the crossover - and quantum critical - regime. This is consistent with the defining property for Fig. 4 (a), namely that the electrical resistivity at $B=0$ is linear in $T$ extending to very low temperatures on the order of $0.1 \mathrm{~K}$. In this temperature regime, the electronic Sommerfeld coefficient, $\gamma \equiv C_{e l} / T$ vs. $T$ at $B=0$ shows essentially the same quantum critical behavior above $T_{\mathrm{N}}$ as its counterpart at $B=B_{c}$ [31]. 
Historically, observing heavy-fermion states using ARPES has been a challenge because their relevant energy scale is very small. Nonetheless, there has been a considerable amount of recent ARPES studies on heavy fermion metals, see below. In particular, ARPES measurements with state-of-art resolution have been carried out in recent years on $\mathrm{YbRh}_{2} \mathrm{Si}_{2}$ at $T>1 \mathrm{~K}$. For a particular part of the Brillouin zone, finite spectral weight was observed for the temperature range from $1 \mathrm{~K}$ up to about $100 \mathrm{~K}$. It is expected that, at even higher temperatures, the lattice Kondo effect should be suppressed and the Fermi surface will be small. The lack of spectral weight at the small Fermi surface for temperatures on the order of $100 \mathrm{~K}$ suggests that, in this ARPES experiment, the matrix element is larger for the $4 f$-electron states than for the conduction-electron states. It would be desirable to carry out ARPES experiments to even higher temperatures in such a way that the small Fermi surface can be observed.

Still, the ARPES experiments by Kummer et al. [19] at $B=0$ are instructive. They reached down to about $1 \mathrm{~K}$. Because at these elevated temperature the system is in the crossover quantum critical regime already at $B=0$, there should be low-energy electronic spectral weight at both the large and the small Fermi surfaces, as discussed above. Thus, ARPES ought to see spectral weight at the large Fermi surface, and it did.

Therefore, the ARPES measurements are consistent with a jump of the Fermi surface as concluded from the low-temperature extrapolation of the isothermal crossovers in magnetotransport and thermodynamic properties (section 4).

\subsection{ARPES in $\mathrm{YbCo}_{2} \mathrm{Si}_{2}$ and other pertinent heavy fermion compounds}

ARPES has become an indispensable tool in investigating the Fermi surface topology of metals and has contributed significantly to our present understanding of $d$-electron based materials in particular, as it gives direct access to the momentum resolved single-electron spectral function. In the context of $f$-electron based compounds like the heavy fermion met-

als with their dynamically generated small energy scales, the utilization of photoemission-based techniques has so far suffered from limited resolution in momentum and energy. In recent years, significant progress in instrumentation has led to an energy and momentum resolution close to the scales relevant for these materials. As a result, a significant number of ARPES studies on heavy fermion metals were recently reported. Besides $\mathrm{YbRh}_{2} \mathrm{Si}_{2}$ 
[19, 32], these include its trivalent counterpart $\mathrm{YbCo}_{2} \mathrm{Si}_{2}$ [33], the antiferromagnets $\mathrm{CeCu}_{2} \mathrm{Ge}_{2}$ [34, $\mathrm{CeRhIn}_{5}$ [35], and the heavy fermion superconductors $\mathrm{Ce}_{2} \mathrm{CoIn}_{8}$ and $\mathrm{Ce}_{2} \mathrm{RhIn}_{8}$ [36, 37]. Several groups have carried out ARPES measurements on $\mathrm{CeCoIn}_{5}$ [38, 39, 40, 41, 42]. A $4 f$-derived band persisting to comparably high temperatures has been reported in [38]. But while the work reported in [39, 40] seems to be more compatible with bandstructure calculations where the $4 f$-electron is treated as localized [40], a more recent study identified a large Fermi surface [41] and discussed the dependence of the result on photon energy. These results point to a moderate to strong $k_{z}$ dependence of the hybridization in line with recent scanning tunneling spectroscopy and optical conductivity measurements [42].

The small energy scales present in the heavy fermion metals lead to good tunability of the ground state with applied magnetic field or pressure. Making use of this tunability within ARPES has so far been a challenge, see below. It thus becomes pertinent to analyze ARPES data of different compounds at ambient conditions within the general phase diagram of the heavy fermion compounds. For $\mathrm{YbRh}_{2} \mathrm{Si}_{2}$, an ARPES study on its trivalent partner $\mathrm{YbCo}_{2} \mathrm{Si}_{2}$ has been reported in Ref. [33]. $\mathrm{YbCo}_{2} \mathrm{Si}_{2}$ possesses an antiferromagnetic ground state formed by localized $4 f$ moments and displays an only moderate mass enhancement as compared to $\mathrm{YbRh}_{2} \mathrm{Si}_{2}$. According to the general phase diagram, when we get deep into the ordered phase, the Fermi surface has to be small [43]. Indeed, this is observed in $\mathrm{YbCo}_{2} \mathrm{Si}_{2}$ together with a weak, band-like feature derived from a crystalline electric field split $4 f$ state [33].

\section{Discussion and Outlook}

Taken together with the anomalous dynamical scaling determined in the critically Au-substituted $\mathrm{CeCu}_{6}$ and the jump of the Fermi surface across the critical pressure found by quantum oscillation experiments in $\mathrm{CeRhIn}_{5}$, the isothermal properties observed across the $T^{*}(B)$ line in the $T-B$ phase diagram of $\mathrm{YbRh}_{2} \mathrm{Si}_{2}$ have been commonly recognized as the Kondo-destruction energy scale expected in the local quantum critical description. As we have emphasized, the $T^{*}(B)$ scale is not only observed by magnetotransport measurements, but is also prominently reflected in thermodynamic quantities.

Unanimous evidence for, or against, a Fermi surface reconstruction across an antiferromagnetic QCP could in principle be obtained by ARPES experiments carried out at sufficiently low temperatures, as function of a non- 
thermal control parameter crossing the QCP. Such experiments have for instance been performed in the high- $T_{c}$ cuprates [44] and the iron pnictides [45, 46] as function of doping and/or chemical pressure. Unfortunately, to detect the signature of the small Fermi surface in $\mathrm{YbRh}_{2} \mathrm{Si}_{2}$ at very low temperatures upon reducing the magnetic field through $B=B^{*}(T \rightarrow 0)$, the needed conditions are unfavorable for ARPES: the temperature must be well below $0.5 \mathrm{~K}$ and the only known tuning parameter is magnetic field. These challenging conditions might, however, be achieved in future quasiparticle interference experiments using scanning tunneling spectroscopy.

As for ARPES experiments on quantum critical heavy fermion systems, in addition to tuning studies using chemical doping/pressure, uniaxial pressure experiments might be a way forward. The challenges for this exciting emerging field, however, remain formidable. To clearly resolve the heavy fermion excitations, further breakthrough in energy resolution and in base temperature is needed. Also the role of surface perfection/termination remains to be further elucidated. ARPES experiments on MBE grown heavy fermion thin films may contribute to further advancing the field.

We thank E. Abrahams, C. Geibel, P. Gegenwart, D. Vyalikh and G. Zwicknagl for useful discussions. We are also grateful to Y. Önuki for generating Fig. 2 from the data of Ref. [11, to P. Gegenwart for supplying the data of Fig. 3(b) [15] and to J. Custers for providing part of Fig. 4(a) [30]. The work has been partially supported by the U.S. Army Research Office Grant No. W911NF-14-1-0497 and the European Research Council Advanced Grant No. 227378 (SP), the German Research Foundation through Forschergruppe 960 (SW and FS), the National Science Foundation of China Grant No. 11474250 (SK), the U.S. Army Research Office Grant No. W911NF-141-0525, NSF Grant No. DMR-1309531 and the Robert A. Welch foundation Grant No. C-1411 (QS).

\section{References}

\section{References}

[1] Special Issue: "Quantum Criticality and Novel Phases" edited by F. Steglich, O. Stockert and S. Wirth, Phys. Status Solidi 247, issue 3 (2010), pages 457764 .

[2] Special Issue: "Quantum Criticality and Novel Phases" edited by S. 
Kirchner, O. Stockert and S. Wirth, Phys. Status Solidi 250, issue 3 (2013), pages 417659.

[3] P. Coleman and A. J. Schofield, Quantum criticality, Nature 433 (2005) 226.

[4] H. von Löhneysen, A. Rosch, M. Vojta and P. Wölfle, Fermi-liquid instabilities at magnetic quantum phase transitions, Rev. Mod. Phys. 79 (2007) 1015.

[5] Q. Si and F. Steglich, Heavy fermions and Quantum Phase Transitions, Science 329 (2010) 1161.

[6] Q. Si and S. Paschen, Quantum phase transitions in heavy fermion metals and Kondo insulators, Phys. Status Solidi 250 (2013) 425.

[7] Q. Si, S. Rabello, K. Ingersent and J. L. Smith, Locally critical quantum phase transitions in strongly correlated metals, Nature 413 (2001) 804.

[8] P. Coleman, C. Pépin, Q. Si and R. Ramazashvili, How do Fermi liquids get heavy and die? J. Phys.: Condens. Matter 13 (2001) R723.

[9] A. Schröder, G. Aeppli, R. Coldea, M. Adams, O. Stockert, H. von Löhneysen, E. Bucher, R. Ramazashvili and P. Coleman, Onset of antiferromagnetism in heavy-fermion metals, Nature 407 (2000) 351.

[10] M. C. Aronson, R. Osborn, R. A. Robinson, J. W. Lynn, R. Chau, C. L. Seaman and M. B. Maple, Non-Fermi-Liquid Scaling of the Magnetic Response in $\mathrm{UCu}_{5-x} \mathrm{Pd}_{x}(x=1,1.5)$, Phys. Rev. Lett. 75 (1995) 725.

[11] H. Shishido, R. Settai, H. Harima, Y. Onuki, A drastic change of the fermi surface at a critical pressure in $\mathrm{CeRhIn}_{5}$ : dHvA study under pressure, J. Phys. Soc. Jpn. 74 (2005) 1103.

[12] T. Park, F. Ronning, H. Q. Yuan, M. B. Salamon, R. Movshovich, J. L. Sarrao and J. D. Thompson, Hidden magnetism and quantum criticality in the heavy fermion superconductor $\mathrm{CeRhIn}_{5}$, Nature 440 (2006) 65.

[13] G. Knebel, D. Aoki, D. Braithwaite, B. Salce and J. Flouquet, Coexistence of antiferromagnetism and superconductivity in $\mathrm{CeRhIn}_{5}$ under high pressure and magnetic field, Phys. Rev. B 74 (2006) 020501. 
[14] S. Paschen, T. Lühmann, S. Wirth, P. Gegenwart, O. Trovarelli, C. Geibel, F. Steglich, P. Coleman and Q. Si, Hall-effect evolution across a heavy-fermion quantum critical point, Nature 432 (2004) 881.

[15] P. Gegenwart, T. Westerkamp, C. Krellner, Y. Tokiwa, S. Paschen, C. Geibel, F. Steglich, E. Abrahams and Q. Si, Multiple energy scales at a quantum critical point, Science 315 (2007) 1049.

[16] S. Friedemann, N. Oeschler, S. Wirth, C. Krellner, C. Geibel, F. Steglich, S. Paschen, S. Kirchner and Q. Si, Fermi-surface collapse and dynamical scaling near a quantum critical point, Proc. Natl. Acad. Sci. USA 107 (2010) 14547.

[17] S. Nair, S. Wirth, S. Friedemann, F. Steglich, Q. Si and A. J. Schofield, Hall effect in heavy fermion metals, Adv. Phys. 61 (2012) 583.

[18] H. Pfau, S. Hartmann, U. Stockert, Peijie Sun, S. Lausberg, M. Brando, S. Friedemann, C. Krellner, C. Geibel, S. Wirth, S. Kirchner, E. Abrahams, Q. Si and F. Steglich, Thermal and electrical transport across a magnetic quantum critical point, Nature 484 (2012) 493.

[19] K. Kummer, S. Patil, A. Chikina, M. Güttler, M. Höppner, A. Generalov, S. Danzenbächer, S. Seiro, A. Hannaske, C. Krellner, Y. Kucherenko, M. Shi, M. Radovic, E. Rienks, G. Zwicknagl, K. Matho, J. W. Allen, C. Laubschat, C. Geibel and D. V. Vyalikh, Temperatureindependent Fermi surface in the Kondo lattice $\mathrm{YbRh}_{2} \mathrm{Si}_{2}$, Phys. Rev. $\mathrm{X}$ 5 (2015) 011028.

[20] J. Hertz, Quantum critical phenomena, Phys. Rev. B 14 (1976) 1165.

[21] A. J. Millis, Effect of a nonzero temperature on quantum critical points in itinerant fermion systems, Phys. Rev. B 48 (1993) 7183.

[22] T. Moriya and T. Takimoto, Anomalous Properties around magnetic Instability in heavy-electron Systems, J. Phys. Soc. Jpn. 64 (1995) 960.

[23] J.-X. Zhu, D. R. Grempel and Q. Si, Continuous quantum phase transition in a Kondo lattice model, Phys. Rev. Lett. 99 (2003) 156404.

[24] Q. Si, J. H. Pixley, E. Nica, S. J. Yamamoto, P. Goswami, R. Yu and S. Kirchner, Kondo destruction and quantum criticality in Kondo lattice systems, J. Phys. Soc. Jpn. 83 (2014) 061005. 
[25] J.-X. Zhu, S. Kirchner, R. Bulla, and Q. Si, Zero-temperature magnetic transition in an easy-axis Kondo lattice model, Phys. Rev. Lett. 99 (2007) 227204.

[26] M. Glossop and K. Ingersent, Magnetic quantum phase transition in an anisotropic Kondo lattice, Phys. Rev. Lett. 99 (2007) 227203.

[27] G. Knebel, J. Buhot, D. Aoki, G. Lapertot, S. Raymond, E. Ressouche and J. Flouquet, Antiferromagnetism and Superconductivity in CeRhIn 5 . J. Phys. Soc. Jpn. 80 (2011) SA001.

[28] T. Westerkamp, Dissertation thesis, Technical University Dresden, Germany, 2008.

[29] S. Friedemann, N. Oeschler, S. Wirth, C. Krellner, C. Geibel, F. Steglich, Q. Si, Discontinuous Hall coefficient at the quantum critical point in $\mathrm{YbRh}_{2} \mathrm{Si}_{2}$. J. Phys.: Condens. Matter, 23(9), (2011) 94216.

[30] J. Custers, P. Gegenwart, H. Wilhelm, K. Neumaier, Y. Tokiwa, O. Trovarelli, C. Geibel, F. Steglich, C. Pépin and P. Coleman, The breakup of heavy electrons at a quantum critical point, Nature 424 (2003) 524 .

[31] N. Oeschler, S. Hartmann, A. P. Pikul, C. Krellner, C. Geibel and F. Steglich, Low-temperature specific heat of $\mathrm{YbRh}_{2} \mathrm{Si}_{2}$, Physica B 403 (2008) 1254 .

[32] S.-K. Mo, W. S. Lee, F. Schmitt, Y. L. Chen, D. H. Lu, C. Capan, D. J. Kim, Z. Fisk, C.-Q. Zhang, Z. Hussain and Z.-X. Shen, Emerging coherence with unified energy, temperature, and lifetime scale in heavy fermion $\mathrm{YbRh}_{2} \mathrm{Si}_{2}$, Phys. Rev. B 85 (2012) 241103(R).

[33] M. Güttler, K. Kummer, S. Patil, M. Höppner, A. Hannaske, S. Danzenbächer, M. Shi, M. Radovic, E. Rienks, C. Laubschat, C. Geibel and D. V. Vyalikh, Tracing the localization of $4 f$ electrons: Angle-resolved photoemission on $\mathrm{YbCo}_{2} \mathrm{Si}_{2}$, the stable trivalent counterpart of the heavy-fermion $\mathrm{YbRh}_{2} \mathrm{Si}_{2}$, Phys. Rev. B 90 (2014) 195138.

[34] M. Y. Kimura, K. Fukushima, H. Takeuchi, S. Ikeda, H. Sugiyama, Y. Tomida, G. Kuwahara, H. Fujiwara, T. Kiss, A. Yasui, I. Kawasaki, H. Yamagami, Y. Saitoh, T. Muro, T. Ebihara and A. Sekiyama, Soft 
x-ray angle-resolved and resonance photoemission study of $\mathrm{CeCu}_{2} \mathrm{Ge}_{2}$ and $\mathrm{LaCu}_{2} \mathrm{Ge}_{2}$, J. Phys.: Conf. Ser. 592 (2015) 012003.

[35] D. P. Moore, T. Durakiewicz, J. J. Joyce, A. J. Arko, L. A. Morales, J. L. Sarrao, P. G. Pagliuso, J. M. Wills and C. G. Olson, The electronic structure of $\mathrm{CeRhIn}_{5}$ and $\mathrm{LaRhIn}_{5}$ from ARPES, Physica B 312-313 (2002) 134 .

[36] S. Raj, Y. Iida, S. Souma, T. Sato, T. Takahashi, H. Ding, S. Ohara, T. Hayakawa, G. F. Chen, I. Sakamoto and H. Harima, Angle-resolved and resonant photoemission spectroscopy on heavy-fermion superconductors $\mathrm{Ce}_{2} \mathrm{CoIn}_{8}$ and $\mathrm{Ce}_{2} \mathrm{RhIn}_{8}$, Phys. Rev. B 71 (2005) 224516.

[37] R. Jiang, D. Mou, C. Liu, X. Zhao, Y. Yao, H. Ryu, C. Petrovic, K.-M. Ho and A. Kaminski, Electronic structure of $\mathrm{Ce}_{2} \mathrm{RhIn}_{8}$ : A twodimensional heavy-fermion system studied by angle-resolved photoemission spectroscopy, Phys. Rev. B 91 (2015) 165101.

[38] A. Koitzsch, S. V. Borisenko, D. Inosov, J. Geck, V. B. Zabolotnyy, H. Shiozawa, M. Knupfer, J. Fink, B. Büchner, E. D. Bauer, J. L. Sarrao and R. Follath, Observing the heavy fermions in $\mathrm{CeCoIn}_{5}$ by angleresolved photoemission, Physica C 460-462 (2007) 666.

[39] A. Koitzsch, S. V. Borisenko, D. Inosov, J. Geck, V. B. Zabolotnyy, H. Shiozawa, M. Knupfer, J. Fink, B. Büchner, E. D. Bauer, J. L. Sarrao and R. Follath, Hybridization effects in $\mathrm{CeCoIn}_{5}$ observed by angleresolved photoemission, Phys. Rev. B 77 (2008) 155128.

[40] A. Koitzsch, I. Opahle, S. Elgazzar, S. V. Borisenko, J. Geck, V. B. Zabolotnyy, D. Inosov, H. Shiozawa, M. Richter, M. Knupfer, J. Fink, B. Büchner, E. D. Bauer, J. L. Sarrao and R. Follath, Electronic structure of $\mathrm{CeCoIn}_{5}$ from angle-resolved photoemission spectroscopy, Phys. Rev. B 79 (2009) 075104.

[41] J. Xiao-Wen, L. Yan, Y. Li, H. Jun-Feng, Z. Lin, Z. Wen-Tao, L. HaiYun, L. Guo-Dong, H. Shao-Long, Z. Jun, L. Wei, W. Yue, D. Xiao-Li, S. Li-Ling, W. Gui-Ling, Z. Yong, W. Xiao-Yang, P. Qin-Jun, W. ZhiMin, Z. Shen-Jin, Y. Feng, X. Zu-Yan, C. Chuang-Tian and Z. XingJiang, Growth, characterization and Fermi surface of heavy fermion




[42] A. Koitzsch, T. K. Kim, U. Treske, M. Knupfer, B. Büchner, M. Richter, I. Opahle, R. Follath, E. D. Bauer and J. L. Sarrao, Band-dependent emergence of heavy quasiparticles in $\mathrm{CeCoIn}_{5}$, Phys. Rev. B 88 (2013) 035124 .

[43] S. J. Yamamoto and Q. Si, Fermi surface and antiferromagnetism in the Kondo lattice: an asymptotically exact solution in $d>1$ dimensions, Phys. Rev. Lett. 99 (2007) 016401.

[44] A. Damascelli, Z. Hussain and Z.-X. Shen, Angle-resolved photoemission studies of the cuprate superconductors, Rev. Mod. Phys. 75 (2003) 473.

[45] S. Thirupathaiah, E. D. L. Rienks, H. S. Jeevan, R. Ovsyannikov, E. Slooten, J. Kaas, E. van Heumen, S. de Jong, H. A. Dürr, K. Siemensmeyer, R. Follath, P. Gegenwart, M. S. Golden and J. Fink, Dissimilarities between the electronic structure of chemically doped and chemically pressurized iron pnictides from an angle-resolved photoemission spectroscopy study, Phys. Rev. B 84 (2011) 014531.

[46] T. Yoshida, I. Nishi, S. Ideta, A. Fujimori, M. Kubota, K. Ono, S. Kasahara, T. Shibauchi, T. Terashima, Y. Matsuda, H. Ikeda and R. Arita, Two-dimensional and three-dimensional Fermi surfaces of superconducting $\mathrm{BaFe}_{2}\left(\mathrm{As}_{1-x} \mathrm{P}_{x}\right)_{2}$ and their nesting properties revealed by angle-resolved photoemission spectroscopy, Phys. Rev. Lett. 106 (2011) 117001. 